\title{
Psycho Social Perspectives on Support for Children with Special Needs in Inclusive Education
}

\author{
Dr. J. Godwin Prem Singh \\ Principal Investigator UGC (MRP), Associate Professor, Dean International Relations, \\ PG. \& Research Department of Social Work, Bishop Heber College (Autonomous), Tiruchirappalli
}

\begin{abstract}
Inclusion of children with special needs with normal children is the provision of least restrictive environment for the differently abled children. This approach helps the differently abled children to grow and develop like normal children. It promotes healthy social relationships between normal and differently abled children and provides equal educational opportunity. It enhances special needs children's growth and development on par with their normal peers. This study aimed at analyse the nature of support services received by children with special needs, intervention facilitated by the schools for enriching the learning environment for CWSN and positive influence of inclusive education for CWSN. The universe of the present study includes all the 191 Government primary schools in 15 blocks of Tiruchirappalli District. From this sampling frame, the researcher selected 4 schools from each block using stratified disproportionate random sampling method. 689 children with special needs are studying in the selected schools. The sample taken for analysis consisted of 300 children with special needs. Out of 60 schools, the researcher selected five children with special needs from each school through stratified disproportionate random sampling method. The study emphasized that promotion of positive attitude towards the differently abled develops healthy social relationships among individuals. Integration helps to raise the differently abled children's standard of living and prepares them to live independently. It reduces psychological problems of disabled children. Basic readiness skills are required to integrate special needs children more effectively. Special and regular school teachers require multi-talents and play diversified roles to handle these children. The researcher also suggests the fact that successful promotion of integration for the children with special needs is achieved certainly if the teachers possess the aforesaid components. Integration warrants whole school approach in which teacher plays a vital role. In an effective inclusive setting, every child will be able to dream his/her own vision of the future and develop abilities to actualize it. The inclusive setting not only increases the involvement of children, but enriches their learning potential. It emphasizes that the child is a human resource in the learning process. The paradigm shift from 'child as a student' to 'child as a human resource' paves the way for a comprehensive and inclusive setting, where every child is treated as special and the teacher becomes a facilitator of learning.
\end{abstract}

Keywords: Inclusive education, Children with Special needs, Achievement Motivation, Social Work Intervention and Attitude.

\section{Introduction}

The belief that education should be available equally to all was also energized by the emergent awareness of individual differences among students and the need, indeed the responsibility of the school, to adapt curricula to these differences. Children with special needs received individualized education programmes with goals, expected learning processes and educational resources tailored to their needs. The movement to individualize education for all children in the context of standards of achievement continues to be one of the central issues in education (Gottlieb, 2003). Philosophies of inclusion and respect for individual differences continue to shape the practice of education and provide the basis for the role of the school social worker. The correspondence between social work values, the emergent mission of education and the role of the school social worker is illustrated by Allen-Meares (1999). The mission of education, implicit in these values, became the basis for school social work in the twentieth century.

School is conceptualized as a community of families and school personnel engaged in the educational process. The educational process is dynamic and wide ranging and involves children, their families, teachers and an institution called school. It is the context for school social work. School is no longer viewed as a building or a collection of classrooms in which teachers and pupils work together (Jenkins, and Rigg, 2003). The school community, no longer simply bounded by geography, comprises all those who engage in the educational process. As in any community, there are varied concrete roles for all members. People fit into these communities in very different ways. Parents and families have membership through their children. Teachers and other school personnel are members with accountability to parents, children and the broader community (Frederickson, \& Cline, 2002). Drawing on each person's capacities, the school social worker focuses on making the educational process work to the fullest extent. Therefore, school social workers work with parents, teachers, pupils, and administrators on behalf of vulnerable children or groups of children. The success of the process depends on involvement of everyone. The social worker helps the school community operate as a real community so that personal, family and community resources can be discovered and used to meet children's developmental needs (Welsh \& Goldberg, 1999). The inclusive education can act as a catalyst for change in educational practice, leading to improved quality of education. Including children with special needs in mainstream schools challenges teachers to develop more child-centered, participatory and active teaching approachesand this benefits all children. However, it promotes activities which help disabled children to develop their full potential, become self-reliant and participate in their communities. At the same time, it challenges discriminatory attitudes in the community, helping parents to think positively about their disabled children and promoting wider social inclusion (Puri, 2008). 


\section{International Journal of Science and Research (IJSR) \\ ISSN (Online): 2319-7064}

Index Copernicus Value (2015): 78.96 | Impact Factor (2015): 6.391

The interventions under SSA for inclusive education are identification, functional and formal assessment, appropriate educational placement, preparation of Individualized Educational Plan, provision of aids and appliances, teacher training, resource support, removal of architectural barriers, research, monitoring and evaluation and a special focus on girls with special needs. The guidelines on inclusive education at SSA ensure that every child with special needs, irrespective of the kind, category and degree of disability, is provided meaningful and quality education. Hence, SSA has adopted a zero rejection policy. This means that no child having special needs should be deprived of the right to education but must be taught in an environment, which is best, suited to his/her learning needs. These include special schools, EGS, AIE or even home-based education. The major thrust of SSA is on inclusion or mainstreaming CWSN into the fabric of formal elementary schooling. Experiences of programmes like DPEP and various research findings have shown that inclusion is best determined by the individual needs of the child (Jambor and Elliott, 2006 Hunt, and Goetz, 1997 and Geetha, 2005). Most children with special needs can be enrolled and retained in regular schools if adequate resource support is provided to them, whereas there are others who might have to be provided some kind of pre-integration programmes, before they can be mainstreamed in a classroom (Nagalakshmi, 2005). There might also be still some CWSN with severe profound disabilities, who may require an educational programme and intensive specialized support completely beyond the purview and scope of a formal school in the current situation (Rudolf and Heredia, 2007). Thus, SSA has adopted a more expansive and broad-based understanding of the concept of inclusion, wherein a multi-option model of educating CWSN is being implemented. The dual objective of embracing this model is to bring more CWSN under the umbrella of SSA and to provide to CWSN appropriate need-based skills, be it vocational, functional literacy or simply activities of daily living (Welsh, \& Goldberg, 1999). Further, an attempt is being made to provide these skills in the most appropriate learning environment. The implementation of this multioption model of inclusion in SSA has been made possible due to the flexibility offered to each State by the programme. Although most SSA States have identified and enrolled CWSN in schools, they differ in the approaches and

Table 1 provides particulars of improvement of the differently abled children. The top four areas where improvement is maximum are: equal educational opportunities (98 percent), improvement in academic standards (96 percent), elimination of discrimination (91 percent) and strengthening of self-confidence ( 89 percent). Education has impact on the children's household chores. Similar were results from the case studies. A majority (Five out of six children with special needs) who participated in the case studies expressed that this inclusive classrooms maximize the opportunity for them to form friendships with students without disabilities. They also pointed out that the normal students agree them as friend and to be members of the class. The results of this study are consistent with those of the study by Rudolf and Heredia, (2007) their study has shown that a majority of the parents have listed a number of improvements in the behaviour of their child at school, home strategies adopted to achieve the ultimate objective of inclusion (Dakar, 2000).

\section{Materials and Methods}

In order to analyze the level of study habit and achievement motivation among the children with special needs, Improvement acquired from inclusive classroom, intervention facilitated by the schools for enriching the learning environment for CWSN and positive influence of inclusive education for CWSW in Tiruchirappalli District, a study on the Social work perspective on inclusive education for children with special needs was conducted. This descriptive study involved Special need children in Government and Government aided primary schools at Tiruchirappalli District. The universe of the present study includes all the 191 Government primary schools in 15 blocks of Tiruchirappalli District. From this sampling frame, the researcher selected 4 schools from each block using stratified disproportionate random sampling method. 689 children with special needs are studying in the selected schools. The sample taken for analysis consisted of 300 children with special needs. Out of 60 schools, the researcher selected five children with special needs from each school through stratified disproportionate random sampling method.

\section{Results and Discussion}

Table 1: Distribution of the respondents according to improvement acquired from inclusive classroom

\begin{tabular}{|c|l|c|c|}
\hline $\begin{array}{c}\text { S. } \\
\text { No. }\end{array}$ & \multicolumn{1}{|l|}{$\begin{array}{c}\text { Improvement acquired from } \\
\text { inclusive classroom }\end{array}$} & $\begin{array}{c}\text { No. of } \\
\text { Respondents* } \\
(\mathrm{n}=224)\end{array}$ & Percentage* \\
\hline 1. & Getting self - reliant & 175 & 78 \\
\hline 2. & Improvement in communication & 168 & 75 \\
\hline 3. & Doing household chores & 75 & 38 \\
\hline 4. & Improvement in studies & 214 & 96 \\
\hline 5. & Overcoming fear about my future & 158 & 71 \\
\hline 6. & Improvement in self-confidence & 200 & 89 \\
\hline 7. & Improvement in mobility & 126 & 56 \\
\hline 8. & Equal educational opportunities & 219 & 98 \\
\hline 9. & $\begin{array}{l}\text { Motivation to compete with } \\
\text { others }\end{array}$ & 187 & 83 \\
\hline 10. & Elimination of discrimination & 203 & 91 \\
\hline
\end{tabular}

* Non-additive frequencies and percents

and social life after being part of IEDC. These findings also have been supported by studies conducted in other countries (Hunt \& Goetz, 1997).

Table 2: Cross table distribution of the gender of the respondents and improvement acquired from the inclusive classroom

\begin{tabular}{|c|l|c|c|c|}
\hline S. No. & Gender & \multicolumn{3}{|c|}{ Improvement Acquired* $(\mathrm{n}=224)$} \\
\hline & & Academic & Psychological & Social \\
\hline 1. & Male & $121(54 \%)$ & $84(38 \%)$ & $113(50 \%)$ \\
\hline 2. & Female & $92(46 \%)$ & $72(32 \%)$ & $88(40 \%)$ \\
\hline
\end{tabular}

*Non-additive frequencies and percents

Cross table 2 revealed that gender has influenced the level of improvement acquired in the inclusive classroom. The male students with special needs have had more improvement in academic ( 54 percent), psychological (38 percent) and social aspects (50 percent). However girl students with special 


\section{International Journal of Science and Research (IJSR) \\ ISSN (Online): 2319-7064 \\ Index Copernicus Value (2015): 78.96 | Impact Factor (2015): 6.391}

needs registered a low level of improvement in academic (46 percent), psychological (32 percent) and social (40 percent) aspects compared with boys. This claim could be further supported by the findings of Katz \& Mirenda, (2002).

Table 3: Cross table distribution of the nature of disability of the respondents and improvement acquired from inclusive classroom

\begin{tabular}{|c|l|c|c|c|}
\hline $\begin{array}{c}\text { S. } \\
\text { No. }\end{array}$ & Nature of disability & \multicolumn{3}{|c|}{ Improvement Acquired* (n=224) } \\
\cline { 2 - 5 } & & Academic & Psychological & Social \\
\hline 1. & Low vision & $18(8 \%)$ & $13(6 \%)$ & $\begin{array}{c}14 \\
(6 \%)\end{array}$ \\
\hline 2. & Hearing Impaired & $22(10 \%)$ & $14(6 \%)$ & $\begin{array}{c}24 \\
(11 \%)\end{array}$ \\
\hline 3. & Physically Impaired & $\begin{array}{c}102 \\
(46 \%)\end{array}$ & $77(34 \%)$ & $\begin{array}{c}95 \\
(42 \%)\end{array}$ \\
\hline 4. & $\begin{array}{l}\text { Mentally Retarded } \\
\text { (Mild) }\end{array}$ & $22(10 \%)$ & $11(5 \%)$ & $\begin{array}{c}21 \\
(9 \%)\end{array}$ \\
\hline 5. & Learning Disability & $49(22 \%)$ & $41(18 \%)$ & $\begin{array}{c}47 \\
(16 \%)\end{array}$ \\
\hline
\end{tabular}

* Non-additive frequencies and percents

From table 3 , it is clear that physically impaired children gain more than children with other disabilities like low vision, poor hearing, mental retardation and learning disability. Studies by Deb, et al., (2002), Wilczenski (1992), Sindelar, et al., (1999) and Bulgren \& Carta, (1998), reveal the fact that physically challenged children show better improvement. The studies also show that most students develop better social skills, self discipline, higher self esteem and greater academic achievement. The teachers were motivated by a determination to diminish or eliminate bullying, teasing or destructive cliques within the school.

Table 4: Distribution of the respondents by various dimensions of study habit

\begin{tabular}{|c|c|c|c|}
\hline S. No & $\begin{array}{l}\text { Various dimensions of } \\
\text { Study habit }\end{array}$ & $\begin{array}{c}\text { No. of } \\
\text { Respondents } \\
(n=300)\end{array}$ & Percentage \\
\hline 1. & $\begin{array}{l}\text { Time management } \\
\text { Low } \\
\text { High }\end{array}$ & $\begin{array}{c}55 \\
245\end{array}$ & $\begin{array}{l}18.3 \\
81.7\end{array}$ \\
\hline 2. & $\begin{array}{l}\text { Reading skill } \\
\text { Low } \\
\text { High }\end{array}$ & $\begin{array}{l}103 \\
197\end{array}$ & $\begin{array}{l}34.3 \\
65.7\end{array}$ \\
\hline 3. & $\begin{array}{l}\text { Listening skill } \\
\text { Low } \\
\text { High }\end{array}$ & $\begin{array}{c}68 \\
232\end{array}$ & $\begin{array}{l}22.7 \\
77.3\end{array}$ \\
\hline 4. & $\begin{array}{l}\text { Concentration } \\
\text { Low } \\
\text { High }\end{array}$ & $\begin{array}{l}159 \\
141\end{array}$ & $\begin{array}{l}53.0 \\
47.0\end{array}$ \\
\hline 5. & $\begin{array}{l}\text { Test anxiety management } \\
\text { Low } \\
\text { High }\end{array}$ & $\begin{array}{c}69 \\
231\end{array}$ & $\begin{array}{l}23.0 \\
77.0\end{array}$ \\
\hline 6. & $\begin{array}{l}\text { School environment } \\
\text { Low } \\
\text { High }\end{array}$ & $\begin{array}{c}93 \\
207\end{array}$ & $\begin{array}{l}31.0 \\
69.0\end{array}$ \\
\hline 7. & $\begin{array}{l}\text { Overall level of study habit } \\
\text { Low } \\
\text { High }\end{array}$ & $\begin{array}{l}105 \\
195\end{array}$ & $\begin{array}{l}35.0 \\
65.0\end{array}$ \\
\hline
\end{tabular}

From the table 4, most students fare very well in time management ( 81.7 percent), listening skill (77.3 percent) and other aspects, but their concentration on study is low (53 percent). Steps may be taken to improve their concentration. For instance, innovative techniques, latest technological devices, the teachers' increased enthusiasm--all these may contribute to the students' concentration. Similar results from the case studies. Mithra, (1998) has studied the relationship between study habit \& academic achievement and have suggested ways and means to improve the two aspects. If, the concentration of the students can be strengthened their performance will surely be better.

Table 5: Distribution of the respondents by various dimensions of motivation

\begin{tabular}{|c|c|c|c|}
\hline S. No & $\begin{array}{c}\text { Various dimensions of } \\
\text { Motivation }\end{array}$ & $\begin{array}{c}\text { No. of } \\
\text { Respondents } \\
(n=300)\end{array}$ & Percentage \\
\hline 1. & $\begin{array}{l}\text { Academic self-concept } \\
\text { Low } \\
\text { High }\end{array}$ & $\begin{array}{c}49 \\
251\end{array}$ & $\begin{array}{l}16.3 \\
83.7\end{array}$ \\
\hline 2. & $\begin{array}{l}\text { Achievement motivation } \\
\text { Low } \\
\text { High }\end{array}$ & $\begin{array}{c}50 \\
250\end{array}$ & $\begin{array}{l}16.7 \\
83.3\end{array}$ \\
\hline 3. & $\begin{array}{l}\text { Social self-concept } \\
\text { Low } \\
\text { High }\end{array}$ & $\begin{array}{c}33 \\
267\end{array}$ & $\begin{array}{l}11.0 \\
89.0\end{array}$ \\
\hline 4. & $\begin{array}{l}\text { Locus of control } \\
\text { Low } \\
\text { High }\end{array}$ & $\begin{array}{c}71 \\
229\end{array}$ & $\begin{array}{l}23.7 \\
76.3\end{array}$ \\
\hline 5. & $\begin{array}{l}\text { Physical Self-concept } \\
\text { Low } \\
\text { High }\end{array}$ & $\begin{array}{c}99 \\
201\end{array}$ & $\begin{array}{l}33.0 \\
67.0\end{array}$ \\
\hline 6. & $\begin{array}{l}\text { Emotional Self-concept } \\
\text { Low } \\
\text { High }\end{array}$ & $\begin{array}{l}137 \\
163\end{array}$ & $\begin{array}{l}45.7 \\
54.3\end{array}$ \\
\hline 7. & $\begin{array}{l}\text { Overall level of motivation } \\
\text { Low } \\
\text { High }\end{array}$ & $\begin{array}{c}82 \\
218\end{array}$ & $\begin{array}{l}27.3 \\
72.7\end{array}$ \\
\hline
\end{tabular}

Table 5 reveals that all the dimensions of motivation are satisfactory such as academic self-concept (83.7 percent), achievement motivation (83.3 percent), social self-concept (89 percent), locus of control (76.3 percent), physical selfconcept (67 percent), emotional self-concept (54.3 percent) and overall level of motivation (72.7 percent). Similar results were arrive at in the study of Montgomery, (2008), Jambor \& Elliott, (2006) and Mrug \& Wallender, (2007) they argued that the mere physical inclusion of the differently abled children in the general classroom does not ensure the children's development, but functional \& social integration is also needed. Teachers should focus attentions strengthening the children's self-concept. They also emphasized that enhancing the self-concept of students has a positive impact on their academic achievements as well as on their personal and social development (Jenkins, and Rigg, 2003). 
International Journal of Science and Research (IJSR)
ISSN (Online): 2319-7064
Index Copernicus Value (2015): 78.96 | Impact Factor (2015): 6.391

Table 6: ' $Z$ ' Test between the gender of the respondents and various dimensions of study habit

\begin{tabular}{|c|c|c|c|c|c|}
\hline S. No & Gender & $\begin{array}{c}\text { Sample Size } \\
\text { (n: 300) }\end{array}$ & $\overline{\mathbf{X}}$ & S.D. & Statistical Inference \\
\hline 1. & $\begin{array}{l}\text { Time management } \\
\text { Male } \\
\text { Female }\end{array}$ & $\begin{array}{l}162 \\
138\end{array}$ & $\begin{array}{l}3.08 \\
3.12\end{array}$ & $\begin{array}{l}1.120 \\
1.088\end{array}$ & $\begin{array}{c}\mathrm{Z}=1.779 \\
\mathrm{P}>0.05 \\
\text { Not Significant }\end{array}$ \\
\hline 2. & $\begin{array}{l}\text { Reading } \\
\text { Male } \\
\text { Female } \\
\end{array}$ & $\begin{array}{l}162 \\
138\end{array}$ & $\begin{array}{l}4.07 \\
4.10 \\
\end{array}$ & $\begin{array}{l}1.386 \\
1.329 \\
\end{array}$ & $\begin{array}{c}\mathrm{Z}=2.449 \\
\mathrm{P}<0.05 \\
\text { Significant }\end{array}$ \\
\hline 3. & $\begin{array}{l}\text { Listening } \\
\text { Male } \\
\text { Female }\end{array}$ & $\begin{array}{l}162 \\
138\end{array}$ & $\begin{array}{l}6.48 \\
6.65\end{array}$ & $\begin{array}{l}1.813 \\
1.628\end{array}$ & $\begin{array}{l}\mathrm{Z}=2.855 \\
\mathrm{P}<0.01 \\
\text { Significant }\end{array}$ \\
\hline 4. & $\begin{array}{l}\text { Concentration } \\
\text { Male } \\
\text { Female }\end{array}$ & $\begin{array}{l}162 \\
138\end{array}$ & $\begin{array}{l}2.20 \\
2.28\end{array}$ & $\begin{array}{l}0.817 \\
0.811\end{array}$ & $\begin{array}{c}\mathrm{Z}=2.773 \\
\mathrm{P}<0.01 \\
\text { Significant }\end{array}$ \\
\hline 5. & $\begin{array}{l}\text { Test anxiety management } \\
\text { Male } \\
\text { Female }\end{array}$ & $\begin{array}{l}162 \\
138\end{array}$ & $\begin{array}{l}4.36 \\
4.60\end{array}$ & $\begin{array}{l}1.377 \\
1.369\end{array}$ & $\begin{array}{c}\mathrm{Z}=0.027 \\
\mathrm{P}>0.05 \\
\text { Not Significant }\end{array}$ \\
\hline 6. & $\begin{array}{l}\text { School environment } \\
\text { Male } \\
\text { Female }\end{array}$ & $\begin{array}{l}162 \\
138\end{array}$ & $\begin{array}{l}8.07 \\
7.97\end{array}$ & $\begin{array}{l}2.665 \\
2.726\end{array}$ & $\begin{aligned} \mathrm{Z}=1.330 \\
\mathrm{P}>0.05 \\
\text { Not Significant }\end{aligned}$ \\
\hline 7. & $\begin{array}{l}\text { Overall level of study habit } \\
\text { Male } \\
\text { Female }\end{array}$ & $\begin{array}{l}162 \\
138\end{array}$ & $\begin{array}{l}28.38 \\
28.52\end{array}$ & $\begin{array}{l}6.952 \\
5.114\end{array}$ & $\begin{array}{l}\mathrm{Z}=2.193 \\
\mathrm{P}<0.05 \\
\text { Significant }\end{array}$ \\
\hline
\end{tabular}

It is clear from the table 6 that there is a significant difference between the gender of the respondents and various dimensions of study habit, such as reading skill $(Z=2.449$, $\mathrm{P}<0.05)$ listening skill $(\mathrm{Z}=2.855, \mathrm{P}<0.01)$, concentration in study $(Z=2.773, P<0.01)$ and overall study habit $(Z=2.193$, $\mathrm{P}<0.05)$. However, there is no significant difference between the gender of the respondents and various dimensions of study habit, like time management, test anxiety management, and school environment. It means that gender has little influence on study habit. The mean score indicates that girls had a more favourable perception of reading skill $(\overline{\mathbf{X}}=4.10)$, listening skill ( $\overline{\mathbf{X}}=6.65)$, concentration in study ( $\overline{\mathbf{X}}=2.28$ ) and overall level of study habit $(\overline{\mathbf{X}}=28.52)$.

Table 7: One way Analysis of Variance among nature of disability of the respondents and selected groups of dimensions

\begin{tabular}{|c|c|c|c|c|c|c|c|}
\hline S. No & Source & $D f$ & $S S$ & $M S$ & \multicolumn{2}{|c|}{$\overline{\mathbf{X}}$} & $\begin{array}{l}\text { Statistical } \\
\text { Inference }\end{array}$ \\
\hline 1. & $\begin{array}{l}\text { School environment } \\
\text { Between Groups } \\
\text { Within Groups }\end{array}$ & $\begin{array}{c}4 \\
295\end{array}$ & $\begin{array}{c}61.921 \\
21339.866\end{array}$ & $\begin{array}{c}15.480 \\
7.254\end{array}$ & $\begin{array}{l}\mathrm{G} 1= \\
\mathrm{G} 2= \\
\mathrm{G} 3= \\
\mathrm{G} 4= \\
\mathrm{G} 5=\end{array}$ & $\begin{array}{l}20.77 \\
19.87 \\
20.61 \\
18.34 \\
19.78\end{array}$ & $\begin{array}{c}\mathrm{F}=2.134 \\
\mathrm{P}>0.05 \\
\text { Not Significant }\end{array}$ \\
\hline 2. & $\begin{array}{l}\text { Fellow students' relationship } \\
\text { Between Groups } \\
\text { Within Groups }\end{array}$ & $\begin{array}{c}4 \\
295\end{array}$ & $\begin{array}{c}57.756 \\
1685.04\end{array}$ & $\begin{array}{c}14.439 \\
5.712\end{array}$ & $\begin{array}{l}\mathrm{G} 1= \\
\mathrm{G} 2= \\
\mathrm{G} 3= \\
\mathrm{G} 4= \\
\mathrm{G} 5=\end{array}$ & $\begin{array}{l}22.92 \\
23.72 \\
25.68 \\
24.19 \\
21.37\end{array}$ & $\begin{array}{c}\mathrm{F}=2.528 \\
\mathrm{P}<0.05 \\
\text { Significant }\end{array}$ \\
\hline 3. & $\begin{array}{l}\text { Parents attitude towards school } \\
\text { Between Groups } \\
\text { Within Groups }\end{array}$ & $\begin{array}{c}4 \\
295\end{array}$ & $\begin{array}{c}33.289 \\
2310.908\end{array}$ & $\begin{array}{l}8.322 \\
7.834\end{array}$ & $\begin{array}{l}\mathrm{G} 1= \\
\mathrm{G} 2= \\
\mathrm{G} 3= \\
\mathrm{G} 4= \\
\mathrm{G} 5=\end{array}$ & $\begin{array}{l}16.87 \\
15.21 \\
16.67 \\
16.54 \\
13.58\end{array}$ & $\begin{array}{c}\mathrm{F}=1.062 \\
\mathrm{P}>0.05 \\
\text { Not Significant }\end{array}$ \\
\hline 4. & $\begin{array}{l}\text { Family adjustment } \\
\text { Between Groups } \\
\text { Within Groups }\end{array}$ & $\begin{array}{c}4 \\
295\end{array}$ & $\begin{array}{c}34.175 \\
756.611\end{array}$ & $\begin{array}{l}8.544 \\
2.565\end{array}$ & $\begin{array}{l}\mathrm{G} 1= \\
\mathrm{G} 2= \\
\mathrm{G} 3= \\
\mathrm{G} 4= \\
\mathrm{G} 5=\end{array}$ & $\begin{array}{l}17.86 \\
16.12 \\
18.39 \\
15.68 \\
17.82\end{array}$ & $\begin{array}{c}\mathrm{F}=3.331 \\
\mathrm{P}<0.05 \\
\text { Significant }\end{array}$ \\
\hline 5. & $\begin{array}{l}\text { Educational adjustment } \\
\text { Between Groups } \\
\text { Within Groups }\end{array}$ & $\begin{array}{c}4 \\
295\end{array}$ & $\begin{array}{c}45.894 \\
1182.636\end{array}$ & $\begin{array}{c}11.473 \\
4.009\end{array}$ & $\begin{array}{l}\mathrm{G} 1= \\
\mathrm{G} 2= \\
\mathrm{G} 3= \\
\mathrm{G} 4= \\
\mathrm{G} 5=\end{array}$ & $\begin{array}{l}16.58 \\
16.78 \\
17.86 \\
15.24 \\
15.98\end{array}$ & $\begin{array}{c}\mathrm{F}=2.861 \\
\mathrm{P}<0.05 \\
\text { Significant }\end{array}$ \\
\hline
\end{tabular}


International Journal of Science and Research (IJSR)

ISSN (Online): 2319-7064

Index Copernicus Value (2015): 78.96 | Impact Factor (2015): 6.391

\begin{tabular}{|c|c|c|c|c|c|c|c|}
\hline$S . N o$ & Source & $D f$ & $S S$ & $M S$ & $\overline{\mathbf{X}}$ & & $\begin{array}{l}\text { Statistical } \\
\text { Inference }\end{array}$ \\
\hline 6. & $\begin{array}{l}\text { Emotional adjustment } \\
\text { Between Groups } \\
\text { Within Groups }\end{array}$ & $\begin{array}{c}4 \\
295\end{array}$ & $\begin{array}{c}67.162 \\
2944.718\end{array}$ & $\begin{array}{c}16.790 \\
9.982\end{array}$ & $\begin{array}{l}\mathrm{G} 1= \\
\mathrm{G} 2= \\
\mathrm{G} 3= \\
\mathrm{G} 4= \\
\mathrm{G} 5=\end{array}$ & $\begin{array}{l}19.58 \\
20.72 \\
20.34 \\
18.79 \\
18.56\end{array}$ & $\begin{array}{c}\mathrm{F}=1.682 \\
\mathrm{P}>0.05 \\
\text { Not Significant }\end{array}$ \\
\hline 7. & $\begin{array}{l}\text { Academic self-concept } \\
\text { Between Groups } \\
\text { Within Groups }\end{array}$ & $\begin{array}{c}4 \\
295\end{array}$ & $\begin{array}{c}245.06 \\
4757.378\end{array}$ & $\begin{array}{l}61.266 \\
16.127\end{array}$ & $\begin{array}{l}\mathrm{G} 1= \\
\mathrm{G} 2= \\
\mathrm{G} 3= \\
\mathrm{G} 4= \\
\mathrm{G} 5=\end{array}$ & $\begin{array}{l}32.56 \\
33.87 \\
33.95 \\
31.99 \\
30.23\end{array}$ & $\begin{array}{c}\mathrm{F}=3.799 \\
\mathrm{P}<0.01 \\
\text { Significant }\end{array}$ \\
\hline 8. & $\begin{array}{l}\text { Achievement Motivation } \\
\text { Between Groups } \\
\text { Within Groups }\end{array}$ & $\begin{array}{c}4 \\
295\end{array}$ & $\begin{array}{c}129.861 \\
4196.976\end{array}$ & $\begin{array}{l}32.465 \\
14.227\end{array}$ & $\begin{array}{l}\mathrm{G} 1= \\
\mathrm{G} 2= \\
\mathrm{G} 3= \\
\mathrm{G} 4= \\
\mathrm{G} 5=\end{array}$ & $\begin{array}{l}28.36 \\
29.45 \\
28.21 \\
28.35 \\
27.89\end{array}$ & $\begin{array}{c}\mathrm{F}=2.281 \\
\mathrm{P}>0.05 \\
\text { Not Significant }\end{array}$ \\
\hline 9. & $\begin{array}{l}\text { Social self-concept } \\
\text { Between Groups } \\
\text { Within Groups }\end{array}$ & $\begin{array}{c}4 \\
295\end{array}$ & $\begin{array}{c}104.062 \\
2902.135\end{array}$ & $\begin{array}{c}26.015 \\
9.838\end{array}$ & $\begin{array}{l}\mathrm{G} 1= \\
\mathrm{G} 2= \\
\mathrm{G} 3= \\
\mathrm{G} 4= \\
\mathrm{G} 5=\end{array}$ & $\begin{array}{l}21.96 \\
20.17 \\
22.98 \\
20.95 \\
19.13\end{array}$ & $\begin{array}{c}\mathrm{F}=2.644 \\
\mathrm{P}<0.05 \\
\text { Significant }\end{array}$ \\
\hline 10. & $\begin{array}{l}\text { Protection } \\
\text { Between Groups } \\
\text { Within Groups }\end{array}$ & $\begin{array}{c}4 \\
295\end{array}$ & $\begin{array}{c}148.363 \\
4105.637\end{array}$ & $\begin{array}{l}37.091 \\
13.917\end{array}$ & $\begin{array}{l}\mathrm{G} 1= \\
\mathrm{G} 2= \\
\mathrm{G} 3= \\
\mathrm{G} 4= \\
\mathrm{G} 5=\end{array}$ & $\begin{array}{l}47.91 \\
48.76 \\
49.00 \\
48.25 \\
46.97\end{array}$ & $\begin{array}{c}\mathrm{F}=2.665 \\
\mathrm{P}<0.05 \\
\text { Significant }\end{array}$ \\
\hline 11. & $\begin{array}{l}\text { Rejection } \\
\text { Between Groups } \\
\text { Within Groups }\end{array}$ & $\begin{array}{c}4 \\
295\end{array}$ & $\begin{array}{c}60.231 \\
3406.449\end{array}$ & $\begin{array}{l}15.058 \\
11.547\end{array}$ & $\begin{array}{l}\mathrm{G} 1= \\
\mathrm{G} 2= \\
\mathrm{G} 3= \\
\mathrm{G} 4= \\
\mathrm{G} 5=\end{array}$ & $\begin{array}{l}58.32 \\
57.23 \\
60.25 \\
60.45 \\
59.65\end{array}$ & $\begin{array}{l}\quad \mathrm{F}=1.304 \\
\mathrm{P}>0.05 \\
\text { Not Significant }\end{array}$ \\
\hline 12. & $\begin{array}{l}\text { Love \& Affection } \\
\text { Between Groups } \\
\text { Within Groups }\end{array}$ & $\begin{array}{c}4 \\
295\end{array}$ & $\begin{array}{c}306.041 \\
7338.039\end{array}$ & $\begin{array}{l}76.510 \\
24.875\end{array}$ & $\begin{array}{l}\mathrm{G} 1= \\
\mathrm{G} 2= \\
\mathrm{G} 3= \\
\mathrm{G} 4= \\
\mathrm{G} 5=\end{array}$ & $\begin{array}{l}59.45 \\
60.33 \\
63.72 \\
62.48 \\
62.87\end{array}$ & $\begin{array}{c}\mathrm{F}=3.076 \\
\mathrm{P}<0.05 \\
\text { Significant }\end{array}$ \\
\hline 13. & $\begin{array}{l}\text { Students attitude towards IE } \\
\text { Between Groups } \\
\text { Within Groups }\end{array}$ & $\begin{array}{c}4 \\
295\end{array}$ & $\begin{array}{c}87.419 \\
1215.328\end{array}$ & $\begin{array}{c}21.855 \\
4.120\end{array}$ & $\begin{array}{l}\mathrm{G} 1= \\
\mathrm{G} 2= \\
\mathrm{G} 3= \\
\mathrm{G} 4= \\
\mathrm{G} 5=\end{array}$ & $\begin{array}{l}28.36 \\
26.54 \\
31.85 \\
30.58 \\
30.21\end{array}$ & $\begin{array}{c}\mathrm{F}=5.305 \\
\mathrm{P}<0.01 \\
\text { Significant }\end{array}$ \\
\hline
\end{tabular}

G1= Low vision G2= Hearing impaired G3 = Physically impaired G4= Mentally retarded (Mild) G5= Learning disability

Table 7 shows that there is a significant variance among nature of disability of the respondents and selected groups of variables, like fellow students' relationship $(\mathrm{F}=2.528$, $\mathrm{P}<0.05)$, family adjustment $(\mathrm{F}=3.331, \mathrm{P}<0.05)$, educational adjustment $(\mathrm{F}=2.861, \quad \mathrm{P}<0.05)$, academic self-concept $(\mathrm{F}=3.799, \mathrm{P}<0.01)$, social self-concept $(\mathrm{F}=2.644, \mathrm{P}<0.05)$, protection $(\mathrm{F}=2.665, \mathrm{P}<0.05)$, love \& affection $(\mathrm{F}=3.076$, $\mathrm{P}<0.05)$ and students' attitude towards inclusive education $(\mathrm{F}=5.305, \mathrm{P}<0.01)$. However, there is no significant variance among the nature of disability of the respondents and selected groups of variables, such as school environment, parents' attitude towards school, emotional adjustment, achievement motivation and rejection. It means that the nature of the disability has influenced the level of perception of various selected predictors perceived by children with special needs. The mean score indicates that different types of differently abled children had different perception of various selected predictors, specifically physically impaired students had higher level of favourable perception of fellow's relationship $(\overline{\mathbf{X}}=25.68)$, family adjustment
( $\overline{\mathbf{X}}=18.39)$, educational adjustment $(\overline{\mathbf{X}}=17.86)$, academic self-concept $(\overline{\mathbf{X}}=33.95)$, social self-concept $(\overline{\mathbf{X}}=22.98)$, protection $(\overline{\mathbf{X}}=49.00)$, love \& affection $(\overline{\mathbf{X}}=63.72)$ and students' attitude towards inclusive education $(\overline{\mathbf{X}}=31.85)$. This result corroborates the findings of Kumar, (2007) who have shown that the normal and the handicapped subjects differed significantly in their home adjustment, social adjustment, and emotional adjustment as well as in overall adjustment. The physically challenged children had a higher level of adjustment in academic, social, family and educational fields. They also argued that the mainstreamed mildly-physically-impaired students reported a higher academic and social efficacy than their non-handicapped.

\section{Implications of the Study}

The present study was conducted to find out positive influence of inclusive education on children with special needs and to identify the problems encounter by children and

\section{Volume 6 Issue 1, January 2017




\section{International Journal of Science and Research (IJSR) \\ ISSN (Online): 2319-7064}

Index Copernicus Value (2015): 78.96 | Impact Factor (2015): 6.391

teacher in the inclusive classroom at Tiruchirappalli district. The present study emphasized that an implementation of the collaborative teaching model brought about various social benefits for groups of students with special needs, they experienced improvement in academic performance, psychological development and social skills; a majority of the students experienced an increase in self-concept related to their social abilities; and accomplishments. The children with special needs who participated in the study noticed that an increase in positive attitude towards themselves and others, a higher degree of motivation and an ability to assess more objectively their skills and weaknesses. The rate of interaction with other students with special needs was a significant predictor of students' educational achievement and thus, it minimizes negative stereotypes on special needs students. This is an important finding since it establishes a clear relationship between social interactions with typical peers and the achievement of individual education plan (IEP) goals by students with special needs. In the present study the interaction and relationship between children with special educational needs and normal peers was high.

The social work intervention is highly essential for those who had low level of perception on these variables. The researcher suggests appropriate social work methods, such as individual intervention, group intervention and community intervention is an essential for solving children problem and make the inclusive classroom is more appropriate to them. The researcher also suggests the following interventions to children. These are: (i) identification of the children with special needs in the classroom; (ii) referring the identified to the experts for further examination and treatment; (iii) developing positive attitude between normal children and children with special needs; (iv) enabling the children with special needs to avail themselves of the facilities provided for them under IED scheme; (v) involving the children with special needs in almost all the activities of the classroom and school and providing scope for cooperative learning among differently abled and normal children through 'play park method'; (vi) parental guidance and counselling and public awareness programme through school activities; (vii) conducting case studies and action research related to the specific problem of children with special needs; (viii) nurturing the talent among children with special needs; and (ix) construction of achievement and diagnostic tool.

Promotion of positive attitude towards the differently abled develops healthy social relationships among individuals. Integration helps to raise the differently abled children's standard of living and prepares them to live independently. It reduces psychological problems of disabled children. Basic readiness skills are required to integrate special needs children more effectively. Special and regular school teachers require multi-talents and play diversified roles to handle these children. Special techniques are used only in the case of children with severe special needs and for developing only the basic academic skills. The mildly disabled do not require such special skills and they need only modifications in the instruction. After learning special skills even the severely differently abled children can enter regular schools with suitable assistive devices. A general teacher has to supervise and facilitate learning of these children in the normal classroom set up. Special educational needs of most of the children with special needs can be met effectively in mainstream schools. The teacher has the responsibility for overall curricular experiences of these children. To fulfil this responsibility the teacher should possess a better awareness of the concept, nature, type and educational requirements to deal with children with special needs. To understand the strengths and weaknesses, every teacher should have a positive attitude towards children with special needs Apart from awareness and attitude a teacher should exhibit specific competencies to plan educational programme for the children with special needs. Successful promotion of integration for the children with special needs is achieved certainly if the teachers possess the aforesaid components. Integration warrants whole school approach in which teacher plays a vital role.

\section{References}

[1] Allen-Meares, P. (1999). The contributions of social workers to schooling-revisited. In. R. Constable, S. McDonald, \& J. Flynn (Eds.), School social work: Practice, policy, and research perspectives (4th ed., pp. 24-31). Chicago: Lyceum Books.

[2] Bulgren, J.A. \& Carta, J.J. (1998). Examining the instructional contexts of students with learning disabilities. Exceptional Children, 59, 182-191.

[3] Dakar, (2000). Frame work for Action: Education for all by 2015. New Delhi (India): Sage publication.

[4] Deb, S., Duggal, C., and Sarkar, A. (2002). Problems Encouraged by Students of Physically Challenged in their Daily School Life and in the Educational Institution. Journal of Disabilities and Impairments, 16 (2), 93-104.

[5] Frederickson, N. \& Cline, T. (2002). Special educational needs, Inclusion and Diversity: A text book. Buckingham: Open University Press.

[6] Geetha, S. (2005). Interventions followed by schools for children with special needs in the inclusive classroom. Unpublished M.Ed. Dissertation, Avinashilingam Deemed University, Coimbatore, Tamilnadu, India.

[7] Gottlieb, J. (2003). Educating children with disabilities in the mainstream. Baltimore: University Park Press.

[8] Heiman, T. (2006). Inclusive schooling: Middle school teachers' perceptions. School Psychology International, 23 (1), 174-186.

[9] Hunt, P. \& Goetz, L. (1997). Research on inclusive educational programs, practices, and outcomes for students with Special Needs. The Journal of Special Education, 31, 3-29.

[10] Jambor, E. Elliott, M. (2006) Self-esteem and Coping Strategies among Deaf Students. Journal of Deaf Studies and Deaf Education, 10, 63-81.

[11] Jenkins, D and Rigg, L.A. (2003). Disability and disadvantage: Selection, Onset and duration effects. Cambridge: Cambridge University Press.

[12] Katz, J. and Mirenda P. (2002). Including Students with Developmental Disabilities in General Education Classrooms: Educational Benefits. International Journal of Special Education, 17(2), 25-19.

[13]Kumar, D. (2007). Adjustment patterns of physically handicapped. Exceptionality, 9, 81-98.

[14] Mithra, A.P. (1998). The relationship between the study habit and the academic achievements. Unpublished 
Ph.D. Thesis, Alagappa University, Karaikudi, Tamilnadu, India.

[15] Montgomery, M. S. (2008) Self-concept and children with learning disabilities: observer-child concordance across six context-dependent domains. Journal of Learning Disabilities, 27, 254-263.

[16] Mrug, S. Wallander, J.L. (2007). Self-concept of Young People with Physical Disabilities: does integration play a role? International Journal of Disability, Development and Education, 49, 267-280.

[17] Nagalakshmi. (2005). Studied an Analysis of Visual Efficiency of Children with Low Vision. Journal of special education, 10 (3), 25-30.

[18] Prakash, R. \& Usha, M.N. (1995). Helping the Disabled: Indian Perspective. New Delhi (India): Ashish Publishing House.

[19] Puri, M. (2008). Handbook of inclusive education for educators, administrator and planners. New Delhi (India): Sage publication.

[20] Rudolf C. Heredia. (2007). Integrated Education for the Disabled Children in Ahmedabad City. Social Action: A Quarterly Review of Social trends, Jan-Mar. Vol. 57(1), Pp 75-83.

[21] Sindelar, P.T., Smith, M.A., Harriman, N.E., Hale, R.L., \& Wilson, R.J. (1999). Teacher effectiveness in special education programmes. Journal of Special Education, 20, 195-207.

[22] Welsh, B. \& Goldberg, G. (1999). Insuring educational success for children-at-risk placed in new learning environments. School Social Work Quarterly, 1(4), 271284.

[23] Wilczenski, F.L. (1992). Measuring attitudes towards inclusive education. Psychology in the Schools, 29, $307-$ 312.

\section{Author Profile}

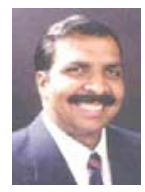

Dr. J. Godwin Prem Singh, is an alumnus of the Department of Social Work, Sacred Heart College, Tirupattur (Vellore District) and has specialized in Human Resource Management. He pursued his M.Phil (Social Work) Degree Course in Organization Behaviour at Madras School of Social Work, Chennai in 1994. In 2001, he acquired the Ph.D. Degree in Social Work from Bharathidasan University. He has also added credit to his academic excellence by acquiring Post Graduate Diplomas in "Business Administration" and "Labour Laws" and P.G. Diploma in Higher Education through Indira Gandhi National Open University, New Delhi. He is a dedicated social work professional (Specialized in Human Resources) with more than 28 years experience of teaching post graduate students. Extensive work in social work children, developing training modules in management, writing course materials and guide \& monitors field work practicum for the PG students. He is service driven, goal oriented, enterprising, with solid integrity, accountability and well organized. Responsible for coordinating and facilitating the administration of Indira Gandhi National Open University, as a Director of Good Samaritan Programme of Bishop Heber College (an Extension activity) and as the Director of CHILDLINE Project of Government of India since Aril 2008 at Bishop Heber College. He is an accepted Research Advisor for the Doctoral Programme of Bharathidasan University, Tiruchirappalli and Martin Luthur University, Shillong, India. He is a member in the High Level Committee of Child Labour Elimination and Effective Rehabilitation, Trichy, constituted by the Collectorate. He visited the Liverpool Hope University to strengthen up the research interaction in Social Sciences discipline and he likely to be there again for a scientific paper presentation in an international convention to be held in July 2009. Currently he has also involved in two researches son child issues (Psycho-Social profile of child workers \& deviant behavior found among Mentally Challenged Children). In 2009, the Global Institute of Economic and Social Development, New Delhi, conferred on him a prestigious award Bharat Shiksha Ratna for his exemplary contribution and achievement made in Education and Social Development. He has also visited Srilanka, UAE and United Kingdom as part of his academic tour while presenting scientific papers. He has received an Educationist award from Indian Council of solidarity, New Delhi and prestegious Mother Teresa Award by Integrated Council of Economic and Social Progress, Kerala State, in 2012. 